\title{
Sonography of Morel-Lavallée Lesions
}

\author{
Colleen Neal, MD, Jon A. Jacobson, MD, \\ Catherine Brandon, MD, Monica Kalume-Brigido, MD, \\ Yoav Morag, MD, Gandikota Girish, MBBS, FRCS, FRCR
}

\begin{abstract}
Objective. The purpose of this series was to retrospectively characterize the sonographic appearance of posttraumatic Morel-Lavallée lesions. Methods. After Institutional Review Board approval was obtained, a search of the radiology information system database with correlation to medical records identified 21 posttraumatic fluid collections of the hip and thigh in 15 patients. Sonographic images were retrospectively reviewed by 1 author to characterize the echogenicity, homogeneity, shape, margins, location, compressibility, and vascularity of the fluid collection. Results were correlated with the age of the fluid collection and aspiration results where possible. Results. All fluid collections (21/21) were located between the deep fat and fascia, with a shape that was fusiform in $12(60 \%)$ of 20 , flat in $5(25 \%)$, and lobular in $3(15 \%)$ (shape not determined in 1 case). Regarding echogenicity, $15(71 \%)$ of the 21 collections were hypoechoic, and 6 (29\%) were anechoic; 13 (62\%) were heterogeneous, and $8(38 \%)$ were homogeneous. The lobular fluid collections were all less than 2 weeks of age, and the flat fluid collections were all greater than 6 months of age. All homogeneous fluid collections were greater than 8 months of age. There was no relationship between the age of a fluid collection and its echogenicity. Conclusions. Morel-Lavallée lesions had a variable appearance, being more homogeneous and flat or fusiform in shape with a well-defined margin as the lesions aged. All Morel-Lavallée lesions were hypoechoic or anechoic, compressible, and located between the deep fat and overlying fascia. Key words: hip; sonography; thigh; trauma.
\end{abstract}

Received January 29, 2008, from the Department of Radiology, University of Michigan Medical Center, Ann Arbor, Michigan USA. Revision requested February 14, 2008. Revised manuscript accepted for publication February 21, 2008.

Address correspondence to Jon A. Jacobson, MD, Department of Radiology, University of Michigan Medical Center, 1500 E Medical Center Dr, TC-2910L, Ann Arbor, MI 481009-0326 USA.

E-mail: jjacobsn@umich.edu

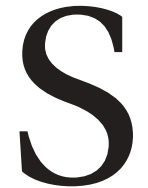

onography can be effectively used to diagnose and characterize soft tissue fluid collections. In the hip region, there is an important type of fluid collection known as the Morel-Lavallée lesion, which is a posttraumatic fluid collection that occurs over the greater trochanter. ${ }^{1}$ A traumatic insult results in the separation of subcutaneous tissue from the underlying fascia with accumulation of blood, lymph, fat, and debris in the potential space..$^{2-4}$ Morel-Lavallée lesions have a tendency to recur and can therefore become chronic sources of pain and infection.

Several case reports and case series have characterized the appearance of Morel-Lavallée lesions by magnetic resonance imaging, showing variable signal characteristics depending on the age of the lesion. ${ }^{3,5-7}$ As the lesion ages, its signal follows that of a seroma: hypointense on T1weighted sequences and hyperintense on T2-weighted sequences. A hypointense capsule frequently surrounds the lesion in all sequences. A limited number of case reports have described the sonographic appearance of Morel-Lavallée lesions as well defined and anechoic., ${ }^{3,4}, 7$ 
At our institution, sonography is frequently used to evaluate posttraumatic fluid collections of the hip. In our clinical experience, we have noted a variable sonographic appearance of MorelLavallée lesions, perhaps related to chronicity, which would affect patient treatment. The purpose of this series was to retrospectively evaluate and characterize the sonographic appearance of Morel-Lavallée lesions.

\section{Materials and Methods}

After Institutional Review Board approval was obtained with informed consent waived, a retrospective search of the radiology information system database was performed. We obtained the sonography reports of 525 patients that described fluid collections of the hip or thigh from January 1998 to April 2006. In addition, 2 patients had sonographic evaluations of the hip and thigh for fluid collections in the 4 months after the database search. Clinical records were then reviewed by 1 of the authors for histories of localized trauma to the hip or thigh region with subsequent development of fluid collections. This review resulted in a total of 21 fluid collections of the hip and thigh in a study group of 15 patients, with 4 patients having sonographic examinations of the same fluid collections at different times.

The sonographic examinations were performed independently by 1 of 10 fellowship-trained musculoskeletal radiologists as part of clinical patient care (range of experience in musculoskeletal sonography, 1-10 years). Static sonograms and cine clips were obtained on one of several clinical ultrasound machines, including iU22 (Philips Medical Systems, Bothell, WA) and LOGIQ 9 (GE Healthcare, Milwaukee, WI) with linear probes (7-12 MHz) and curvilinear probes (5-7 MHz), all with compound imaging.

The static sonograms and cine clips were retrospectively reviewed by 1 of the authors (fellowship-trained musculoskeletal radiologist with 10 years of musculoskeletal sonography experience). Fluid collections were characterized with regard to echogenicity (anechoic, hypoechoic, isoechoic, or hyperechoic compared with surrounding muscle), homogeneity (homogeneous or heterogeneous), shape (flat, fusiform, or lobu- lar), margins (smooth or irregular), and location (within subcutaneous fat, at the fat-muscle fascial border, or intramuscular).

If a cine clip was available, the compressibility of the fluid collection was recorded. If color or power Doppler imaging was present, flow was characterized as present or absent. The largest dimension of the fluid collection was also recorded where possible. Clinical records were reviewed for histories of fluid collection aspirations or surgeries and subsequent physical or pathologic information about the fluid.

\section{Results}

The study group consisted of 11 male and 4 female patients (age range, 17-84 years; mean age, 56 years). Two patients had their fluid collections imaged 3 times, and 2 others had their fluid collections imaged twice, giving a total of 21 posttraumatic fluid collections of the hip and thigh imaged with sonography. The time from the initial trauma to sonography varied among the patients, ranging from 1 week to 4 years (mean, 13 months).

All fluid collections (21/21) were located between the deep fat and fascia over the hip and thigh (Figures 1-4). The average largest dimension of the fluid collections was $8 \mathrm{~cm}$ (range, 3-17 $\mathrm{cm})$. All fluid collections (12/12) that had cine clips were compressible, and all (11/11) with color or power Doppler imaging did not have internal vascularity.

Regarding the shape of the fluid collections, 12 $(60 \%)$ of 20 were fusiform (Figures 2 and 3); 5 (25\%) were flat (Figure 4); and 3 (15\%) were lobular (Figure 1). The lobular fluid collections were all less than 2 weeks of age, and the flat fluid collections were all greater than 6 months of age. In 1 patient, the shape of the fluid collection could not be retrospectively determined because of the sonographic field of view.

Regarding the echogenicity of the fluid collections relative to the surrounding tissue, 15 (71\%) of 21 collections were hypoechoic (Figures 1 and 2), and 6 (29\%) were anechoic (Figures 3 and 4). There was no relationship between the age of a fluid collection and its echogenicity. For example, anechoic fluid collections ranged from 0.5 to 42 months after trauma. 
Regarding the homogeneity of the fluid collections, 13 (62\%) of 21 collections were heterogeneous (Figures 1-3), and 8 (38\%) were homogeneous (Figure 4). The ages of the heterogeneous fluid collections ranged from 1 week to 8 months. All homogeneous fluid collections were greater than 8 months of age.

Regarding margins, 15 (71\%) of 21 collections had smooth margins (Figures 3 and 4), and 6 (29\%) had irregular margins (Figures 1 and 2). The smooth fluid collections were all greater than 18 months of age.

Of the 21 fluid collections, 4 were aspirated. Cytologic evaluation of these aspirates did not reveal malignancy or infection. None of the patients went on to surgery.

\section{Discussion}

Our results show that Morel-Lavallée lesions have a characteristic location between the deep fat and overlying fascia with no relationship between the age of the lesions and their echogenicity because all appeared hypoechoic or anechoic. The acute and subacute lesions had a heterogeneous appearance with irregular margins. The chronic lesions (>18 months) were homogeneous with smooth margins. Overall, fluid collections of less than 1 month of age tended to be lobular, and chronic fluid collections of several months or older were more likely flat or fusiform in shape. All fluid collections were compressible without flow on color Doppler imaging.

A Morel-Lavallée lesion results from the traumatic separation of subcutaneous tissue from the underlying fascia, specifically over the greater trochanter. ${ }^{1}$ Morel-Lavallée lesions most commonly develop secondarily to blunt trauma and are associated with pelvic and acetabular fractures. ${ }^{1}$ Morel-Lavallée lesions are also known as closed internal degloving injuries. ${ }^{2}$ Separation between the fascia and subcutaneous tissue creates a potential space that fills with blood, lymph, fat, and other debris. The clinical presentation includes bruising, a fluctuant soft tissue mass, skin hypermobility, and decreased cutaneous sensations. ${ }^{2}$ The lesion may develop slowly and therefore may be missed on the initial posttraumatic evaluation. A local inflammatory reaction can result in formation of a fibrous

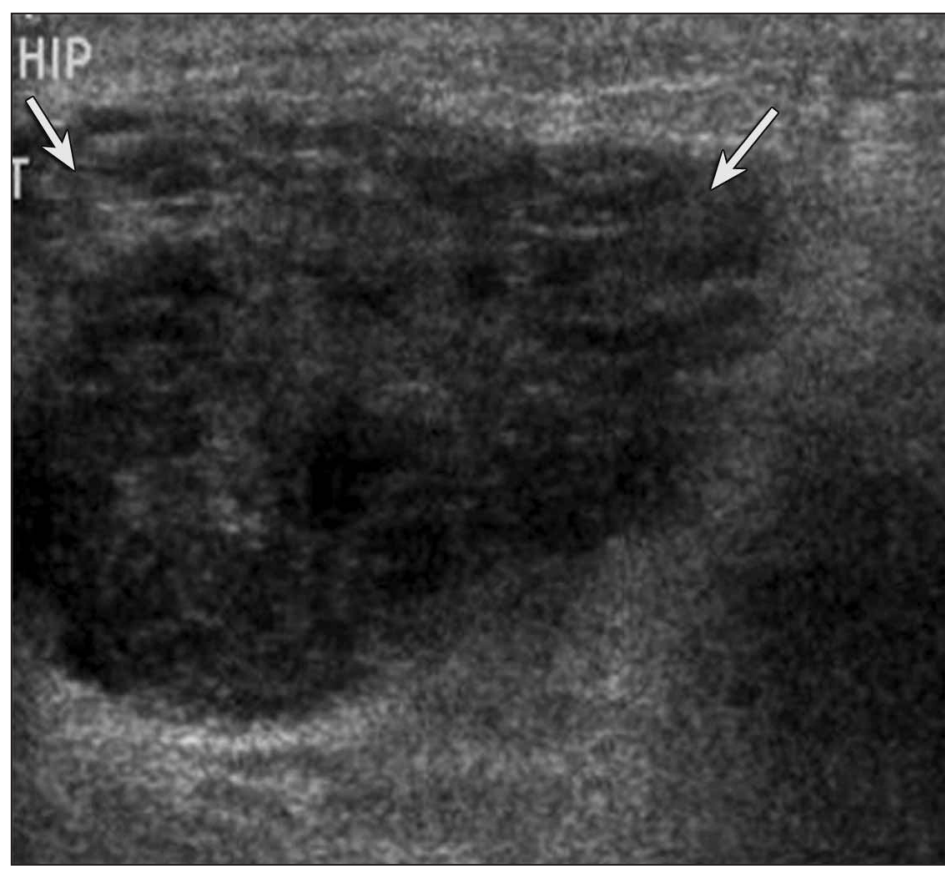

Figure 1. Sonogram from a 63-year-old male patient 2 weeks after injury showing a lobular hypoechoic and heterogeneous fluid collection (arrows) with irregular margins and without flow on color Doppler imaging (not shown).

Figure 2. Color Doppler sonogram from a 51-year-old female patient 3 weeks after injury showing a fusiform predominantly hypoechoic and heterogeneous fluid collection (arrows) with irregular margins and no intrinsic vascularity. The fluid collection was compressible (not shown).

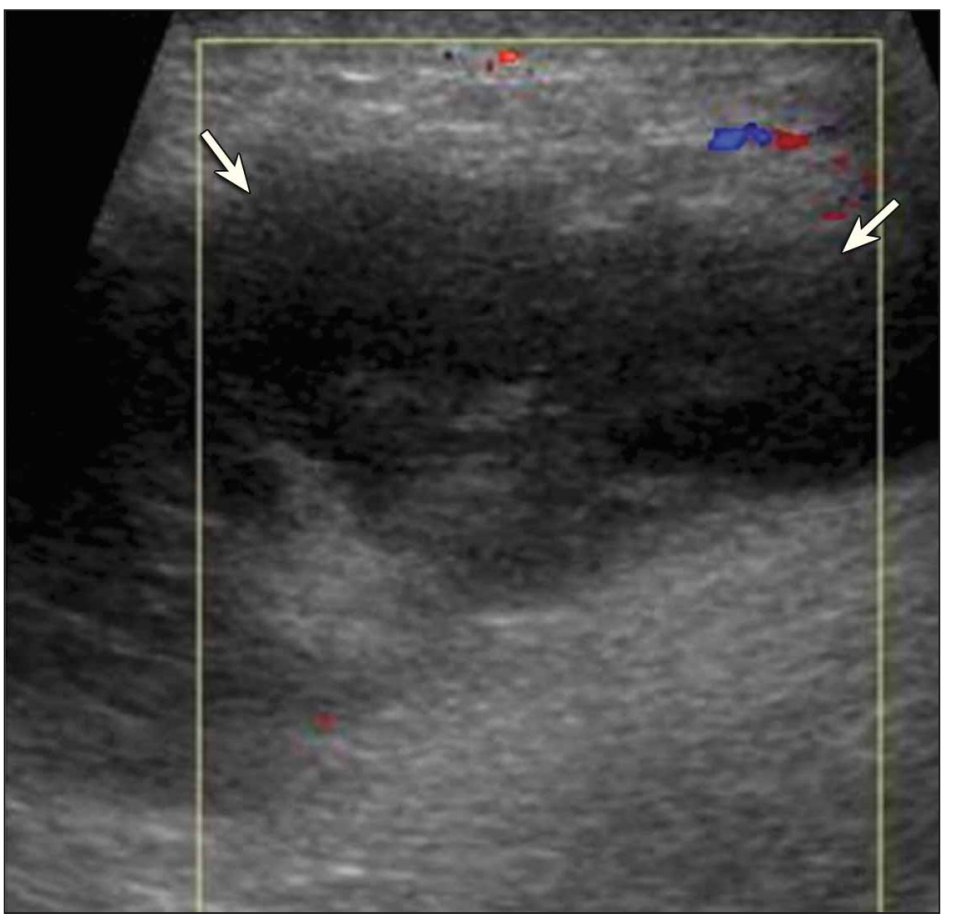




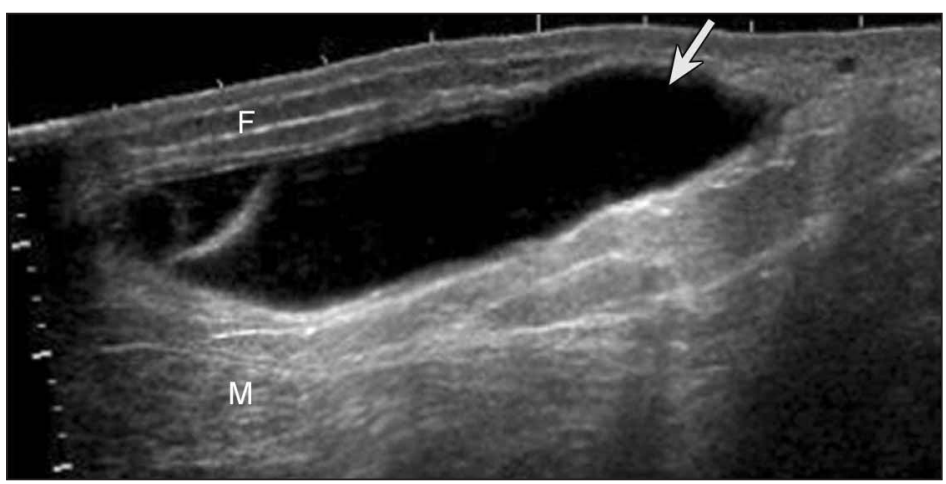

Figure 3. Extended field-of-view sonogram from a 17-year-old male patient 3 months after injury showing a fusiform heterogeneous but predominantly anechoic fluid collection (arrow) with smooth margins, which was compressible and had no intrinsic flow on color Doppler imaging (not shown). F indicates fat; and M, muscle.

pseudocapsule as the lesion ages, ${ }^{3,7}$ contributing to the lesion's propensity to persist and recur. Early diagnosis and treatment reduce the risk of the lesion's becoming a chronic source of pain and potential infection. If a lesion is small and acute without a pseudocapsule, a complete resolution may be achieved with conservative management such as compression. ${ }^{4,7}$ Percutaneous aspiration with suction drainage has been used with success ${ }^{9}$; however, for larger lesions, many orthopedic surgeons favor early surgical drainage and debridement. ${ }^{2}$ Fluoroscopic percutaneous talc sclerodesis has been proposed as a method to prevent fluid reaccumulation after drainage. ${ }^{10}$

Figure 4. Sonogram from an 84-year-old male patient 16 months after injury showing a flat anechoic and homogeneous fluid collection (arrow) with smooth margins located between the subcutaneous fat and adjacent musculature, which was compressible (not shown).

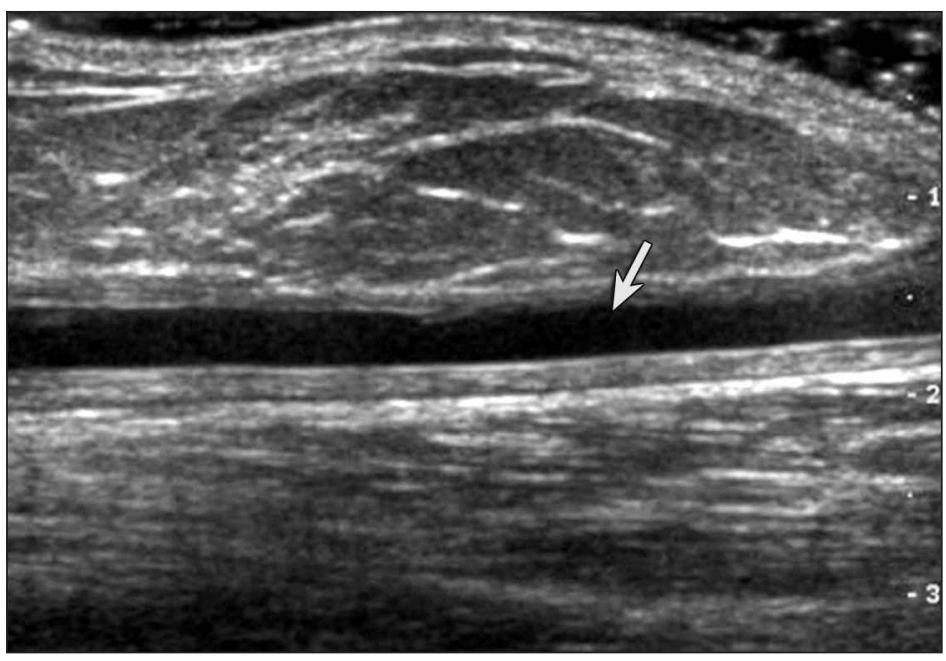

The sonographic findings of Morel-Lavallée lesions are concordant with their known pathophysiologic mechanisms. Early, the cavity fills with blood, lymph, and fat. ${ }^{2-4}$ In our series, these lesions were irregularly marginated and tended to be heterogeneous. As the lesion evolves, blood is reabsorbed and replaced with serosanguinous fluid, ${ }^{3,6}$ contributing to the tendency of these lesions to become more homogeneous as they age. However, the lesions may have repeated hemorrhage, ${ }^{2}$ which may contribute to the lack of correlation we found between age and echogenicity. For example, a chronic lesion may show increased echogenicity if it has rebled. Additionally, a MorelLavallée lesion may have fat remnants both peripherally and within the fluid collection, ${ }^{4,6-8}$ which is another factor that may contribute to the lack of correlation between a lesion's age and its echogenicity. Finally, we found the Morel-Lavallée lesions to be more well defined and smoothly marginated as they became more chronic, which may have been due in part to the tendency of these lesions to form pseudocapsules. ${ }^{3,7}$

The sonographic characteristics of MorelLavallée lesions are somewhat variable. In our study, lesions of less than 1 month of age tended to be heterogeneous and irregularly marginated. There is a wide range of diagnoses for such a lesion, including abscesses, hematomas, fat necrosis, and neoplasms. Although the consistencies of a posttraumatic hematoma and an acute Morel-Lavallée lesion are similar, the Morel-Lavallée lesion has a specific anatomic location and pathophysiologic process that make it more prone to complications and chronicity. As the Morel-Lavallée lesions evolved, we found that they became more regularly marginated and homogeneous. Similarly, there is a wide series of differential diagnoses for such lesions, including seromas, bursitis, lymphoceles, and neoplasms.

The limitations of this series included those inherent to a retrospective sonographic study. We relied on the sonographer to image all pertinent findings, which may have been affected by the skill and experience of the sonographer. Another limitation of this study was the lack of a reference standard with which to compare our sonographic findings because 4 patients had negative cytologic findings from aspirates, and none had surgery; 
however, all patients had histories of trauma and development of fluid collections characteristic of Morel-Lavallée lesions. In addition, although the ages of the lesions were calculated from the dates of injury, we could not exclude the possibility of intermittent or recurrent fluid accumulations within these intervals. Finally, the small sample of 21 Morel-Lavallée lesions was a relative limitation.

In summary, although acute Morel-Lavallée lesions tended to be heterogeneous and lobular with irregular margins, their sonographic appearance became more homogeneous and flat or fusiform in shape with a well-defined margin as the lesions aged. All Morel-Lavallée lesions were hypoechoic or anechoic, compressible, and located between the deep fat and overlying fascia.

\section{References}

1. Letournel E, Judet R. Fractures of the Acetabulum. 2nd ed. New York, NY: Springer-Verlag; 1993:337.

2. Hak DJ, Olson SA, Matta JM. Diagnosis and management of closed internal degloving injuries associated with pelvic and acetabular fractures: the Morel-Lavallée lesion. J Trauma 1997; 42:1046-1051.

3. Puig J, Pelaez I, Baños J, et al. Long-standing MorelLavallée lesion in the proximal thigh: ultrasound and MR findings with surgical and histopathologic correlation. Australas Radiol 2006; 50:594-597.

4. Parra JA, Fernandez MA, Encinas B, Rico M. Morel-Lavallée effusions in the thigh. Skeletal Radiol 1997; 26:239-241.

5. Gilbert BC, Bui-Mansfield LT, Delong S. MRI of a MorelLavallée lesion. AJR Am J Roentgenol 2004; 182:13471348.

6. Mellado JM, Pérez del Palomar L, Díaz L, Ramos A, Saurí A. Long-standing Morel-Lavallée lesions of the trochanteric region and proximal thigh: MRI features in five patients. AJR Am J Roentgenol 2004; 182:1289-1294.

7. Mukherjee K, Perrin SM, Hughes PM. Morel-Lavallée lesion in an adolescent with ultrasound and MRI correlation. Skeletal Radiol 2007; 36(suppl 1):S43-S45.

8. Kalaci A, Karazincir S, Yanat AN. Long-standing MorelLavallée lesion of the thigh simulating a neoplasm. Clin Imaging 2007; 31:287-291.

9. Tseng S, Tornetta P III. Percutaneous management of Morel-Lavallée Lesions. J Bone Joint Surg Am 2006; 88:9296.

10. Luria S, Applbaum Y, Weil $Y$, Liebergall M, Peyser A. Talc sclerodesis of persistent Morel-Lavallée lesions (posttraumatic pseudocysts): case report of 4 patients. J Orthop Trauma 2006; 20:435-438. 